\title{
The Nigrostriatal Tract between the Substantia Nigra and Striatum in the Human Brain: A Diffusion Tensor Tractography Study
}

\author{
Sang Seok Yeo, Jeong Pyo Seo \\ Department of Physical Therapy, College of Health Sciences, Dankook University, Cheonan, Republic of Korea
}

\begin{abstract}
Objectives: The nigrostriatal tract (NST) connect from the substantia nigra pars compacta to the striatum. A few previous studies have reported on the NST in the Parkinson's disease using a proboblistic tractography method. However, no study has been conducted for identification of the NST using streamline DTT technique. In the current study, we used streamline DTI technique to investigate the reconstruction method and characteristics of the NST in normal subjects.

Methods: Eleven healthy subjects were recruited in this study. The NST from the substantia nigra of the midbrain and the striatum of basal ganglia was reconstructed using DTI data. Fractional anisotropy, apparent diffusion coefficient (ADC) values and fiber numbers of the NST were measured.

Results: In all subjects, the NST between the substantia nigra of the midbrain and the striatum. Mean values for FA, ADC, and tract volume were $0.460,0.818$, and 154.3 in the right NST, and $0.485,0.818$, and 176.3 in the left NST respectively.

Conclusions: we reconstructed the NRT from the substantia nigra of the midbrain and the striatum of the basal ganglia using streamline tractography method. We believe that the findings and the proposed streamline reconstruction method of this study would be useful in future researches on the NST of the human brain.
\end{abstract}

Keywords: Diffusion tensor imaging, Diffusion tensor tractography, Nigrostriatal tract, Dopaminergic pathways

\section{INTRODUCTION}

The nigrostriatal tract (NST), which is one of the major bilateral dopaminergic pathways that link the brainstem to the striatum, connects from the substantia nigra pars compacta in the midbrain to the dorsal striatum. ${ }^{1-3}$ It is major dopaminergic pathways in the human brain, and belong the basal ganglia motor loop which is critical as part of a system in the production of movement. ${ }^{4}$ The degeneration of the NST is led to be the cause of the motor symptoms of Parkinson's disease including tremors, rigidity, hypokinesia, and postural imbalance. ${ }^{1,5}$

Recent developments in diffusion tensor tractography (DTT), derived from diffusion tensor imaging (DTI), have allowed for the three dimensional visualization and localization of the various neural tracts in the human brain. ${ }^{6-8} \mathrm{~A}$ few previous studies have reported on the NST in the Par-

Received Dec 9, 2020 Revised Dec 16, 2020

Accepted Dec 17, 2020

Corresponding author Jeong Pyo Seo

E-mail raphael0905@hanmail.net kinson's disease using a proboblistic tractography method. ${ }^{3,9}$ Although a streamline DTI technique is a powerful anatomic imaging tool in a clinical field and it can be applied in native space, without registration to a template, ${ }^{10,11}$ no study has been conducted for identification of the NST using streamline DTT technique.

In the current study, we used streamline DTI technique to investigate the reconstruction method and characteristics of the NST in normal subjects.

\section{METHODS}

\section{Subjects}

Eleven right-handed healthy subjects (males: 7, females: 4, mean age: 29.2 years, range: 20-38 years) with no previous history of neurological, physi-

Copylight (C)2020 The Korean Society of Physical Therapy

This is an Open Access article distribute under the terms of the Creative Commons Attribution Non-commercial License (https:// creativecommons.org/license/by-nc/4.o.) which permits unrestricted non-commercial use, distribution, and reproduction in any medium, provided the original work is properly cited. 
cal, or psychiatric illness were recruited for this study. All subjects understood the purpose of this study and provided written, informed consent prior to participation. The study protocol was approved by our local Institutional Review Board.

\section{Data acquisition}

A 6-channel head coil on a 1.5 T Philips Gyroscan Intera (Philips, Ltd, Best, The Netherlands) with single-shot echo-planar imaging was used for acquisition of DTI data. For each of the 32 gradients, 70 contiguous slices were acquired parallel to the anterior commissure-posterior commissure line. Imaging parameters of DTI were as follows: acquisition matrix $=96 \times$ 96; reconstructed to matrix $=192 \times 192$; field of view $=240 \times 240 \mathrm{~mm}^{2}$; repetition time $=10,398 \mathrm{~ms}$; echo time $=72 \mathrm{~ms}$; parallel imaging reduction factor $=2$; echo-planar imaging factor $=59 ; b=1,000 \mathrm{~s} / \mathrm{mm}^{2}$; number of excitations $=1$; and a slice thickness of $2.5 \mathrm{~mm}$.

\section{Fiber tracking}

Eddy current-induced image distortions were removed using affine multiscale two-dimensional registration in the Oxford Centre for Functional Magnetic Resonance Imaging of Brain (FMRIB) Software Library (FSL; www.fmrib.ox.ac.uk/fsl). ${ }^{12}$ DTI-Studio software (CMRM, Johns Hopkins Medical Institute, Baltimore, MD, USA) was used to evaluate NSTs. ${ }^{13}$ For reconstruction of the NST, a first region of interest (ROI) was manually drawn on the substantia nigra on the FA map at midbrain, and a second ROI was placed on the striatum on the FA map., ${ }^{3,14}$ In a nigrofugal tracing study, traversing of nigrostriatal projections from the globus pallidus to the striatum was demonstrated. Moreover, the NST from the posterior putamen have been found to form a discrete bundle coursing through the globus pallidus to converge at the substantia nigra. ${ }^{3}$ Fiber tracking was started at the center of a seed voxel with a fractional anisotropy (FA) of $>0.15$ and ended at a voxel with a fiber assignment of $>0.15$ and a tract turning-angle of $<70^{\circ}$, with an option of cut operation on the axial images. ${ }^{12} \mathrm{FA}$, apparent diffusion coefficient (ADC) values and fiber numbers of the NST were measured in both hemispheres.

\section{Statistical analysis}

SPSS software version 15.0 (Chicago, SPSS Inc.) was used for the analysis. An independent t-test was used for determination of variances in the value of FA, ADC, and fiber numbers of the NST between the right and left hemispheres. Statistical significance was accepted for $\mathrm{p}$ values of $<0.05$.

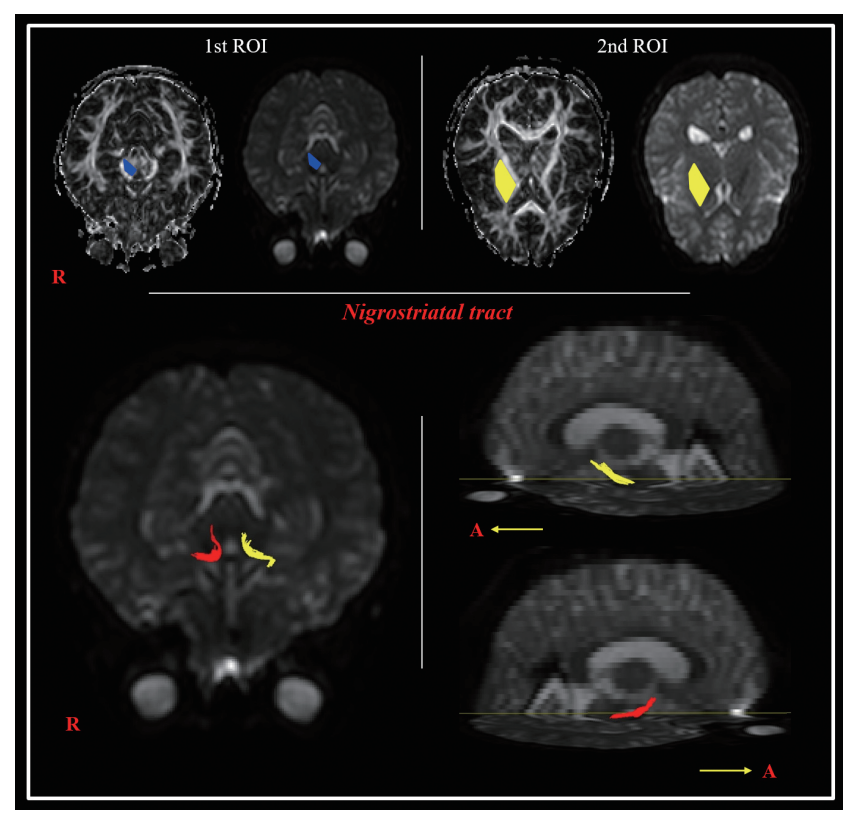

Figure 1. The region of interest (ROI) and results of diffusion tensor tractography for the the nigrostriatal tract. The first region of interest (ROI) was placed on the substantia nigra (blue), and the second ROI was placed on the striatum (yellow). The NST are reconstructed in both hemispheres. (20-year-old female).

Table 1. Diffusion tensor tractography parameters of the nigrostriatal tract

\begin{tabular}{lccc}
\hline Hemisphere & FA & ADC & Fiber number \\
\hline Right & $0.460( \pm 0.039)$ & $0.818( \pm 0.072)$ & $154.3( \pm 49.7)$ \\
Left & $0.485( \pm 0.054)$ & $0.818( \pm 0.095)$ & $176.4( \pm 57.6)$ \\
Total & $0.473( \pm 0.048)$ & $0.864( \pm 0.082)$ & $165.8( \pm 53.7)$ \\
\hline
\end{tabular}

Values indicate mean ( \pm standard deviation).

FA: fractional anisotropy, ADC: apparent diffusion coefficient.

\section{RESULTS}

In all subjects, the NST between the substantia nigra of the midbrain and the striatum were reconstructed (Figure 1). Mean values for FA, ADC, and fiber number were $0.460,0.818$, and 154.3 in the right NST, and 0.485 , 0.818 , and 176.3 in the left NST respectively. No significant differences were observed in FA, ADC, and fiber number between the both hemispheres in the NST $(\mathrm{p}>0.05)$ (Table 1$)$.

\section{DISCUSSION}

In the current study, the NST in the normal human brain was reconstructed between the substantia nigra of the midbrain and the striatum, using the streamline tractography technique. Determination of ROIs is 
fundamental to analysis for the DTT. We selected ROIs for reconstruction of NST: the substantia nigra on the midbrain, and the striatum. We found that the NST originated from the substantia nigra on the midbrain and terminated in the striatum of basal ganglia. Therefore, we believe the reconstruction of this neural tract to be precise and reproducible using streamline tractography technique.

Since introduction of DTT, a few studies DTT studies have been conducted on the NST in the Parkinson's disease. ${ }^{3,9}$ Andica et al. ${ }^{3}$ reported on degeneration of the NST in the patients with Parkinson's disease using generate tractography. In 2008, Camp et al. ${ }^{9}$ investigated that the substantia nigra and did not include the nigrostriatal fiber tract in the Wistar rats brain. However, these studies described the reconstruction of the NST using probabilistic tractography method. The probabilistic tractography takes into account intra-voxel crossing fibers, estimates the pathways which originate at seed voxel and provides quantitative information about the probability of structural connectivity that a white matter tract in the human brain. ${ }^{15}$ The stream line tractography method is the most intuitive way to reconstruct a $3 \mathrm{D}$ trajectory from a $3 \mathrm{D}$ vector field by following the local vector orientation, and it has advantage in simplifying visualization considering medical application. ${ }^{16,17}$ To the best of our knowledge, this is the first DTT study to reconstruct the NST in human brain using streamline tractography method. However, limitations of DTI should be considered. DTI may underestimate fiber tracts, due to crossing fiber or partial volume effect, and it is difficult to reflect all fibers, particularly small fibers. ${ }^{18,19}$

In conclusion, we reconstructed the NST from the substantia nigra of the midbrain and the striatum of the basal ganglia using streamline tractography method. We believe that the findings and the proposed streamline reconstruction method of this study would be useful to neuroscience clinicians in clinical practice and future researches on the NST of the human brain. Conduct of further complementary studies involving larger case numbers is warranted. In addition, we suggest further studies are required for the clinical studies which are related with the NST injury.

\section{REFERENCES}

1. Burke RE, O’Malley K. Axon degeneration in Parkinson's disease. Exp Neurol. 2013;246:72-83.
2. Cheng HC, Ulane CM, Burke RE. Clinical progression in Parkinson disease and the neurobiology of axons. Ann Neurol. 2010;67(6):715-25.

3. Andica C, Kamagata K, Hatano T et al. Neurite orientation dispersion and density imaging of the nigrostriatal pathway in Parkinson's disease: Retrograde degeneration observed by tract-profile analysis. Parkinsonism Relat Disord. 2018;51:55-60.

4. Ikemoto S. Brain reward circuitry beyond the mesolimbic dopamine system: a neurobiological theory. Neurosci Biobehav Rev. 2010;35(2): 129-50.

5. Nieuwenhuys R, Voogd J, Huijzen CV. The human central nervous system: a synopsis and atlas. New York, Springer, 2008;807-40.

6. Jang SH, Kwon HG. Relationship between depression and dorsolateral prefronto-thalamic tract injury in patients with mild traumatic brain injury. Sci Rep. 2020;10(1):1-6.

7. Jang SH, Kwon YH. The relationship between consciousness and the ascending reticular activating system in patients with traumatic brain injury. BMC Neurol. 2020;20(1):375.

8. Jang SH, Seo YS. Recovery of Injured Optic Radiations in a Patient with Hypoxic-Ischaemic Brain Injury. Neuro Ophthalmol. 2019;44(4):270-3.

9. Van Camp N, Blockx I, Verhoye M et al. Diffusion tensor imaging in a rat model of Parkinson's disease after lesioning of the nigrostriatal tract. NMR Biomed. 2009;22(7):697-706.

10. Assaf Y, Pasternak O. Diffusion tensor imaging (DTI)-based white matter mapping in brain research: a review. J Mol Neurosci. 2008;34(1):51-61.

11. Ressel V, Van Hedel HJA, Scheer I et al. Comparison of DTI analysis methods for clinical research: influence of pre-processing and tract selection methods. Eur Radiol Exp. 2018;2(1):33.

12 Smith SM, Jenkinson M, Woolrich MW et al. Advances in functional and structural MR image analysis and implementation as FSL. Neuroimage. 2004;23:S208-19.

13 Jiang H, Van Zijl PCM, Kim J et al. Dtistudio: Resource program for diffusion tensor computation and fiber bundle tracking. Comput Methods Programs Biomed. 2006;81(2):106-16.

14. Song YJC, Korgaonkar MS, Armstrong LV et al. Tractography of the brainstem in major depressive disorder using diffusion tensor imaging. PLoS one. 2014;9(1):e84825.

15.Behrens TEJ, Woolrich MW, Jenkinson M et al. Characterization and propagation of uncertainty in diffusion-weighted MR imaging. Magn Reson Med. 2003;50(5):1077-88.

16. Mori S, Van Zijl PCM. Fiber tracking: principles and strategies - a technical review. NMR Biomed. 2002;15(7-8):468-80.

17. Clark CA, Barrick TR, Murphy MM et al. White matter fiber tracking in patients with space-occupying lesions of the brain: a new technique for neurosurgical planning? Neuroimage 2003;20(3):1601-8.

18 Parker GJ, Alexander DC. Probabilistic anatomical connectivity derived from the microscopic persistent angular structure of cerebral tissue. Philos Trans R Soc Lond B Biol Sci. 2005;360(1457):893-902.

19 Yamada K. Diffusion tensor tractography should be used with caution. Proc Natl Acad Sci U S A. 2009;106(7):E14. 